\title{
Systematic analysis of the p53-related microRNAs in breast cancer revealing their essential roles in the cell cycle
}

\author{
ENXIANG ZHOU ${ }^{1}$, NA HUI ${ }^{2}$, MIN SHU ${ }^{2}$, BAIPING WU $^{1}$ and JIANLIN ZHOU ${ }^{2}$ \\ ${ }^{1}$ Department of General Surgery, The Second Xiangya Hospital, Central South University, Changsha, Hunan 410011; \\ ${ }^{2}$ Key Laboratory of Protein Chemistry and Developmental Biology of the Ministry of Education, College of Life Science, \\ Hunan Normal University, Changsha, Hunan 410081, P.R. China
}

Received November 8, 2014; Accepted September 4, 2015

DOI: $10.3892 / \mathrm{ol} .2015 .3751$

\begin{abstract}
Numerous miRNAs have been found to be involved in the regulation of the p53 signaling pathway. Conversely, p53 regulates the transcription or processing of microRNAs (miRNAs). Given that complexities in the association between p53 and miRNAs exist, and due to the rapidly increasing amount of literature regarding the interactions between p53 and miRNAs, the present study systematically analyzed the associations between miRNAs and p53 in breast cancer using a literature-based discovery approach, natural language processing. A total of 22 miRNAs were found to be associated with p53. Next, three popular online tools (PicTar, miRanda and TargetScan) were used to predict the targets of each miRNA, and certain targets were validated by experiments. Gene Ontology annotation and network analysis demonstrated that the majority of the targets of the p53-related miRNAs were enriched in the cell cycle process. These results suggest that, in addition to regulating the transcription of cell cycle-related genes, p53 also indirectly modulates the cell cycle via miRNAs.
\end{abstract}

\section{Introduction}

Tumor suppressor p53 plays a central role in protecting cells against carcinogenesis, mainly functioning as a transcription factor. In response to stress signals, such as DNA damage, oncogenic stimuli and hypoxia, the p53 protein regulates the transcription of numerous different genes, leading to cell

Correspondence to: Professor Enxiang Zhou, Department of General Surgery, The Second Xiangya Hospital, Central South University, 139 Renmin Middle Road, Changsha, Hunan 410011, P.R. China

E-mail: zhouenxiang@medmail.com.cn

Professor Jianlin Zhou, Key Laboratory of Protein Chemistry and Developmental Biology of the Ministry of Education, College of Life Science, Hunan Normal University, 36 Lushan Road, Changsha, Hunan 410081, P.R. China

E-mail: jlzhou@hunnu.edu.cn

Key words: natural language processing, microRNA, p53, breast cancer, cell cycle cycle arrest, apoptosis, DNA repair or senescence (1). The inactivation of $\mathrm{p} 53$ by mutation is a frequent event in carcinogenesis. Mutations in the TP53 gene, which encodes the p53 protein, occur in around half of all tumor specimens, but the overall frequency of p53 mutations in breast cancer is only $20-30 \%(2,3)$. It is believed that, in breast cancer harboring the wild-type p53 gene, p53 function is compromised by other genetic or epigenetic alterations (4,5). A number of studies have demonstrated that changes in interactome components or the target genes of p53 could contribute to reduce the roles of p53 during stress [reviewed in $(4,5)]$.

Recently, a number of microRNAs (miRNAs) have been found to be involved in the p53 signaling pathway and breast carcinogenesis (6). Certain miRNAs directly target the mRNA of p53 and negatively regulate p53 expression, such as miR-125b (7), miR-375 (8) and miR-504 (9). A study in murine models of postmenopausal breast cancer suggested that miR-504 expression induced by obesity contributes to the reduced 553 protein expression and mammary tumor progression (9). Another class of miRNAs indirectly affected p53 signaling through regulating genes associated with p53. For example, miR-21 antagonizes the p53 pathway in breast cancer by inhibiting the expression of p53-regulated genes (10); oncomiRs miR-221/222 promoted proliferation in breast cancer by inhibiting p53 upregulated modulator of apoptosis expression (10). Conversely, p53 can regulate miRNA transcription, for example, that of miR-10b (11), miR-22 (12), miR-26a (13), miR-34a (14), miR-148a (15), miR-200b (16), miR-200c (16) and miR-205 (17), or miRNA processing, such as that of miR-16 $(13,18)$, miR-145 $(18,19)$ and miR-203 $(20)$.

Given the rapidly increasing amount of literature regarding the interaction between p53 and miRNAs, and as complexities in the association between p53 and miRNAs exist, the present study systematically analyzed p53-related miRNAs and their targets in breast cancer using a literature-based discovery approach, natural language processing (NLP).

\section{Materials and methods}

NLP analysis of miRNAs associated with p53 and breast cancer. NLP analysis was performed as described by Gao et al (21) and Tang et al (22). Briefly, a PubMed search was conducted with the following combination of query terms: 
('mammary cancer' OR ‘mammary tumour' OR 'mammary tumor' OR 'mammary neoplasm' OR 'mammary carcinoma' OR 'breast cancer' OR 'breast tumour' OR 'breast tumor' OR 'breast neoplasm' OR 'breast carcinoma') AND ('p53' OR 'TP53' OR 'TRP53'). All the miRNAs reported in each of the studies were compiled in a list, and then subjected to gene mention tagging using A Biomedical Named Entity Recognizer, an open source tool for automatically tagging genes, proteins and other entity names in text (23). For conjugated terms, conjunction resolution was performed to obtain individual terms, for example, 'miR-200b/c' was resolved into 'miR-200b' and 'miR-200c'. In the present study, all the genes and miRNAs were named using the official symbol in the Entrez and miRBase databases, respectively. Finally, the co-citation frequency of each miRNA with p53 and breast cancer in the PubMed abstracts was calculated as described by Gao et al (21). The higher the co-citation frequency of a miRNA with p53 and breast cancer, the closer it is associated with p53 and breast cancer.

Prediction of miRNA targets. The targets of the miRNAs were predicted using the following computational programs: PicTar 2005 (24) (http://pictar.mdc-berlin.de/cgi-bin/PicTar_vertebrate.cgi), miRanda v5 (25) (http://www.ebi.ac.uk/enright-srv/ microcosm/htdocs/targets/v5) and TargetScan 5.1 (26) (http://www.targetscan.org).

Analysis of gene ontology (GO), pathways and networks. Go analysis was performed with GSEABase package from $\mathrm{R}$ statistical platform (http://www.r-project.org/). Genes were categorized based on biological process (BP), molecular function (MF) and cellular component (CC). GenMAPP v2.1 was used to map genes to the Kyoto Encyclopedia of Genes and Genomes (KEGG) pathway database, and calculate the enrichment $\mathrm{P}$-value for each pathway (27).

Network analysis of miRNA targets. To construct gene interaction networks, the following three different interaction associations were integrated: i) Protein interaction, gene regulation and protein modification in the KEGG database; ii) high-throughout protein interaction experiments such as yeast two-hybrid experiments; and iii) gene interaction associations that have previously been reported. Pathway data were downloaded from the KEGG database and used to analyze the interaction associations of genes [including enzyme-enzyme associations, protein-protein interactions (PPIs) and gene expression interactions] with the KEGGSOAP package (http://www.bioconductor.org/packages/2.4/bioc/html/KEGGSOAP. html). The PPI data were downloaded from the MIPS database (http://mips.helmholtz-muenchen.de/proj/ppi/) (28). For the interactions that have been reported, the co-citation frequency of each gene pair in the PubMed abstracts was calculated as described by Gao et al (21). Finally, all three types of data were integrated and mapped in a network structure using Medusa (29).

Construction of miRNA target expression plasmid. Each pair of complementary oligonucleotides containing the predicted miRNA target region were synthesized, annealed and ligated into pmirGLO Dual-Luciferase miRNA target expression vectors (Promega, Madison, WI, USA) at NheI/SalI sites. To ensure that the overhangs created by oligonucleotide annealing were complementary to the two ends of linearized vector, CTAG (protruding sequence of NheI digestion) and TCGA (protruding sequence of SalI digestion) were added to the 5 ' ends of the forward and reverse oligonucleotides, respectively. For clone confirmation, a KpnI restriction site was added to each pair of oligonucleotides. When digested with $K p n \mathrm{I}$, the correct construct releases an $\sim 500$-bp insert due to a $K p n I$ site at position 478 in the vector. All the plasmids were further confirmed by DNA sequencing.

Cell culture, transfection and luciferase assay. Cell culture, transfection and luciferase assays were performed as previously described (30). Human embryonic kidney 293 (HEK293) cells (American Type Culture Collection, Manassas, VA, USA) were cultured on 24-well plates, and co-transfected with 10 pmol miRNA mimics (GenePharma, Shanghai, China) and $0.4 \mu \mathrm{g}$ miRNA target expression plasmids. At 24-h post-transfection, the cells were harvested and assayed for luciferase activity using the Dual-Glo luciferase assay system (Promega). The Firefly luciferase activities were normalized to Renilla luciferase activity. The relative Firefly luciferase activity of the cells transfected with miRNA mimics was represented as the percentage of activity relative to that of the cells transfected with negative control miRNA mimics. For each transfection, the luciferase activity was averaged from three replicates.

Statistical analysis. The Student's t-test was used to evaluate statistical significance and all statistical analyses were performed using $\mathrm{R}$ project statistical software (http:// www.r-project.org/). $\mathrm{P}<0.05$ was considered to indicate a statistically significant difference.

\section{Results}

Identification of miRNAs associated with 53 and breast cancer by NLP analysis. NLP has been successfully used to identify molecular interactions. To find the miRNA interacting with p53 in breast cancer, the present study searched PubMed with the following combination of query terms: ('mammary cancer' OR 'mammary tumour' OR 'mammary tumor' OR 'mammary neoplasm' OR ‘mammary carcinoma' OR ‘breast cancer' OR 'breast tumour' OR 'breast tumor' OR 'breast neoplasm' OR 'breast carcinoma') AND ('p53' OR 'TP53' OR 'TRP53'), and obtained 5,525 studies reporting on p53 and breast cancer. Further analysis, as described in the Materials and methods section, identified 22 miRNAs that are reported to interact with p53 in breast cancer (Table I). Among these miRNAs, the three most frequently cited were miR-34a, miR-21 and miR-200c, which were cited by 8,6 and 5 studies, respectively.

Computational prediction and experimental investigation of miRNA targets. To make a reliable prediction, three popular online tools (PicTar, miRanda and TargetScan) were used to predict the targets of each p53- and breast cancer-related miRNA. These tools make predictions based on different features of miRNA-mRNA interactions (31). Therefore, for a certain miRNA, the three tools provide different lists of predicted targets. The common targets predicted by these 
Table I. p53- and breast cancer-related miRNAs and their predicted targets.

\begin{tabular}{|c|c|c|}
\hline miRNA & $\begin{array}{l}\text { PubMed } \\
\text { count }\end{array}$ & Predicted targets \\
\hline $\operatorname{miR}-34 a$ & 8 & $\begin{array}{l}\text { ZNF281, RPS6KA4, PNOC, SYVN1, MYRIP, CRHR1, TAF5, MPP2, CACNB3, DPYSL4, EVI5L, } \\
\text { STRN3, UHRF2, AXL, COPS7B, ACSL4, ASB1, SNX15, ALDOA }\end{array}$ \\
\hline miR-21 & 6 & $\begin{array}{l}\text { WWP1, NFIB }, \text { CCL1, C17ORF39, NTF3, ASPN, CNTFR, PELI1, SOX2, JAG1, RECK, TGFBI, } \\
\text { MATN2, SPRY2 }\end{array}$ \\
\hline miR-200c & 5 & DGKA, BAP1, NDST1 \\
\hline $\operatorname{miR}-200 b$ & 4 & HS3ST1 \\
\hline miR-200a & 3 & SPAG9, DIXDC1, GATA6, TCERG1, HMG20A, TP53INP1, SOX5, PCDH9 \\
\hline miR-203 & 3 & $\begin{array}{l}\text { COPS7B, PHLDA3, CCNG1, DLG5, DGKZ, } \underline{\text { CITED2 }}, \underline{\text { GLI3 }}, \text { DUSP5, DLX } 5, \text { ACO2, ARNTL, } \\
\text { FOXK2, } \underline{\text { CUL1 }}, \text { C18ORF } 34, \text { CSN2, OVOL1 }, \text { ZNF281, GABRB2 }\end{array}$ \\
\hline miR-205 & 3 & $\begin{array}{l}\text { DLG2, E2F1, C21ORF63, LRP1, HS3ST1, ERBB3, PHC2, FRK, ADAMTS9, INHBA, } \\
\text { INPPL1, IPO7 }\end{array}$ \\
\hline miR-145 & 2 & $\begin{array}{l}\text { GLIS1, SEMA3A, FKBP3, NEDD9, ATXN2, C11ORF9, SLITRK4, CCNL1, ZBTB10, PLCL2, } \\
\text { RGS7, RTKN, CTNNBIP1, LENG8, SEMA6A, ZNF423, ACTG1, ARPC5, SRGAP1 }\end{array}$ \\
\hline $\operatorname{miR}-155$ & 2 & $\begin{array}{l}\text { SALL1, IKBKE, SDCBP, HIVEP2, BOC, H3F3A, FBXO11, ACTA1, BRD1, LRP1B, CARHSP1, } \\
\text { SOCS1, TP53INP1, WEE1, RNF123, MYO10, DNAJB7, AICDA, ASTN2, CSNK1G2, CHD7, } \\
\text { MAP3K10, CSF1R, HBP1, CEBPB }\end{array}$ \\
\hline $\operatorname{miR}-10 b$ & 1 & DOCK11, HS6ST2, CECR6, NCOR2, FXR2, ARIH2, DAZAP1 \\
\hline $\operatorname{miR}-133 \mathrm{a}$ & 1 & $\begin{array}{l}\text { SOLH, PTHR1, CTBP2, ATP6AP2, RAPH1, CSNK1G3, RCE1, CLTA, EVI1, ELF2, TFAP2D, } \\
\text { MLLT3, VPS54, NRIP3, PTPRD, LRRC7, NDRG1, ABCA2, GDI2 }\end{array}$ \\
\hline miR-148a & 1 & $\begin{array}{l}\text { ATP6AP2, ITSN2, ROBO1, GTF2H1, YPEL3, USP47, KLF4, AKAP1, ABCB7, RAB34, CNTN4, } \\
\text { WNT10B, ALS2CR2, SFRS11, NOG, PRKAG2, MTF1, GAP43, CCKBR, SYNJ1, MAF1, } \\
\text { GPR116, C1GALT1, GADD45A, DYRK1B, TRPS1, DNMT1, PLAA, SFRS2IP, UCP3, MLLT10, } \\
\text { USP48, LBR, CHD7, COL2A1, RNF38 }\end{array}$ \\
\hline miR-16 & 1 & 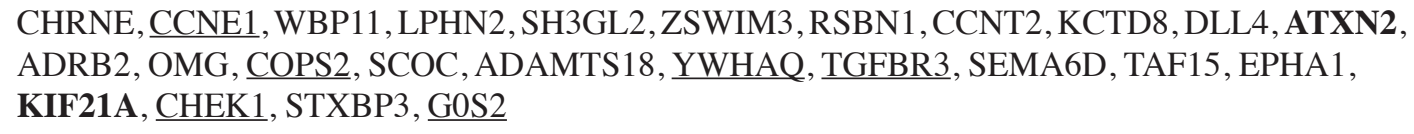 \\
\hline miR-191 & 1 & RNF139, GAP43, PLCD1, NDST1 \\
\hline $\operatorname{miR}-210$ & 1 & EFNA3 \\
\hline $\operatorname{miR}-22$ & 1 & $\begin{array}{l}\text { JMJD1 A, PURB, ERBB3, ODF1, CSF1R, MAX, EMILIN3, SATB2, IPO7, PRR6, RFXANK, } \\
\text { SV2A, EPC1, STAG2, TRUB1, FAM49B, MTHFD2, IL13RA1, DNAJB5, TAGLN, CAV3, } \\
\text { CDKN1A, MECP2, ZFYVE9, NFYA, BCL9L, MAT2A }\end{array}$ \\
\hline $\operatorname{miR}-222$ & 1 & RBM24, CDKN1C, GNAI2, IRX5, KHDRBS2, RSBN1L, CDKN1B, MESDC1 \\
\hline $\operatorname{miR}-26 a$ & 1 & 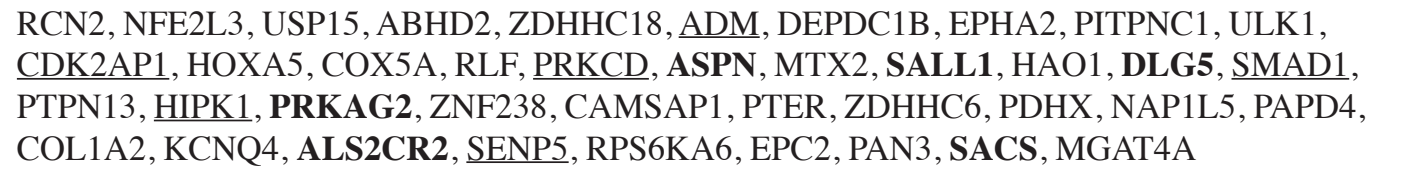 \\
\hline miR-9 & 1 & $\begin{array}{l}\text { CCNE2, ENTPD5, FOXP4, NOX4, ONECUT1, RNF111, RBM9, RPS6KA4, DYRK1B, ITPKC, } \\
\text { CNTFR, PYGO2, GAD1, RAB34, ARPC1A, SLC35B3, ODZ1, PARG, SACS, FBXW2, FBN1, } \\
\text { AUH, ARMCX2, LEPRE1, PLSCR3, LRCH4, MUM1L1, KIF21A, ERBB2IP, CALB2, TNFRSF21, } \\
\text { CTHRC1, TBPL1, EVI5L, NCOR2, TESK2, SLC30A3, HDAC5, ARID1A, SLC31A2, RANBP2, } \\
\text { SLC27A4, DHX40, AP2M1, PCSK6, LAMP1, PALMD, NID2, CSDA, DBNL, DIAPH1, SLC10A3, } \\
\text { SNX7, LMNA, TGOLN2, P4HA2, TRIM2, AP3B1, LHFP }\end{array}$ \\
\hline
\end{tabular}

$\operatorname{miR}-342$

$\operatorname{miR}-497$

miR-504

1

Common targets of two or more miRNAs are shown in bold. The underlined genes belong to GO term 'cell cycle and proliferation'. GO, Gene Ontology; miRNA, microRNA.

three prediction tools were chosen for further analysis in the present study. With the exception of miR-342, miR-497 and miR-504, each miRNA exhibited a different number of predicted targets. The miRNA with the most targets was miR-9, with 59 targets, while miR-200b and miR-210 only had one target. A total of 320 genes were predicted to be targeted 


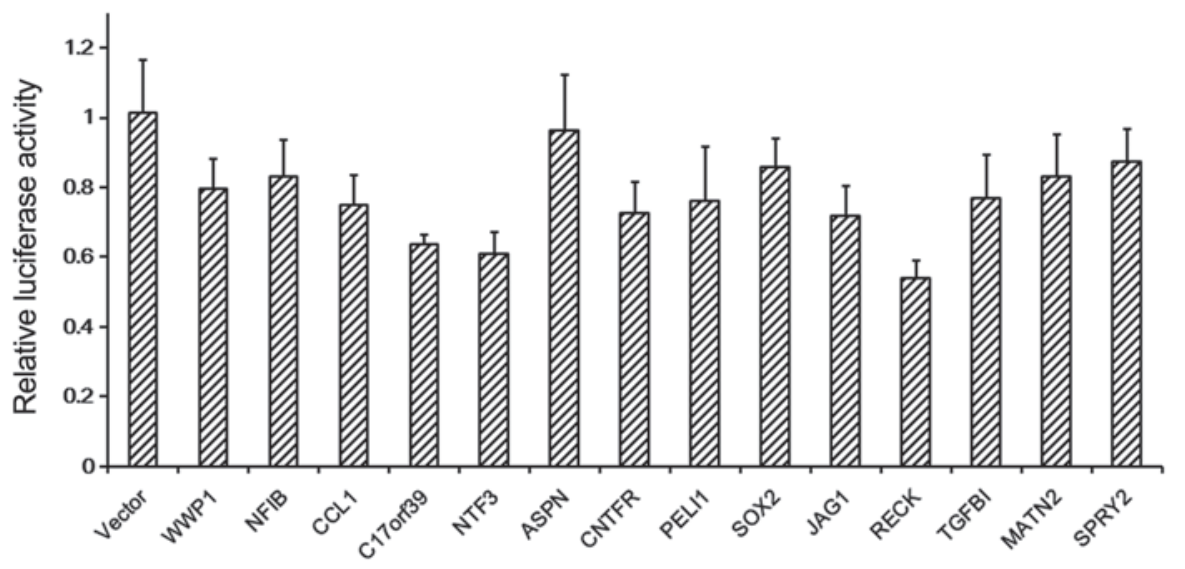

Figure 1. Repression of firefly luciferase by the interaction between miR-21 and its predicted binding site. HEK293 cells were co-transfected with miRNA mimics and miRNA target expression plasmids. At 24-h post-transfection, the cells were harvested and assayed for luciferase activity. The firefly luciferase activities were normalized to Renilla luciferase activity. The relative firefly luciferase activity of the cells transfected with miRNA mimics is represented as the percentage of activity relative to that of the cells transfected with negative control miRNA mimics. Data are shown as the mean \pm standrad deviation of three independent experiments. miR/miRNA, microRNA.

Table II. Top 5 significantly enriched Gene Ontology terms in the microRNA targets.

\begin{tabular}{llrl}
\hline Category & \multicolumn{1}{c}{ Term } & $\mathrm{n}$ & P-value \\
\hline CC & Nucleus & 108 & $1.67 \times 10^{-7}$ \\
& Extracellular matrix & 14 & $2.98 \times 10^{-4}$ \\
& ER/golgi & 23 & 0.347901 \\
& Plasma membrane & 47 & 0.351261 \\
& Cytosol & 6 & 0.530201 \\
MF & Transcription regulatory activity & 40 & $9.53 \times 10^{-7}$ \\
& Kinase activity & 30 & $3.15 \times 10^{-5}$ \\
& Extracellular structural activity & 3 & 0.007524 \\
& Enzyme regulator activity & 19 & 0.009502 \\
& Nucleic acid binding activity & 56 & 0.010738 \\
BP & Cell cycle and proliferation & 42 & $5.36 \times 10^{-6}$ \\
& RNA metabolism & 70 & $3.13 \times 10^{-5}$ \\
& Cell organization and biogenesis & 50 & $4.74 \times 10^{-4}$ \\
& Protein metabolism & 57 & 0.027053 \\
Cell death & 21 & 0.031232 \\
& & &
\end{tabular}

MF, molecular function; $\mathrm{CC}$, cellular component; $\mathrm{BP}$, biological process.

by these 19 miRNAs (Table I). Among these, 25 genes were able to be targeted by two miRNAs.

To validate the above prediction, the pmirGLO Dual-Luciferase miRNA target expression vector was used to investigate whether miR-21 could bind to its targets as predicted. Each of the 14 predicted miR-21 binding sites was cloned downstream of the Firefly luciferase of the pmirGLO vector, and co-transfected with miR-21 or scramble mimics into HEK293 cells. Luciferase assay showed that $13 / 14$ of the predicted targets of miR-21 (not the ASPN gene) could be regulated by miR-21 (Fig. 1). Moreover, 8/14 of the predicted miR-21 targets have been validated by other studies [WWP1 (32), NFIB (33), PELI1 (34), SOX2 (35), JAG1 (36), RECK (37), TGFBI (36)
Table III. Kyoto Encyclopedia of Genes and Genomes pathways overrepresented in the lists of microRNA targets.

\begin{tabular}{lrc}
\hline Pathway & $\mathrm{n}$ & P-value \\
\hline Cell cycle & 11 & $3.83 \times 10^{-5}$ \\
Axon guidance & 9 & 0.002147584 \\
p53 signaling pathway & 6 & 0.003804563 \\
Notch signaling pathway & 4 & 0.019950756 \\
Phosphatidylinositol signaling & 5 & 0.026477865 \\
system & & \\
Hedgehog signaling pathway & 4 & 0.037347722 \\
TGF- $\beta$ signaling pathway & 5 & 0.041980514 \\
Basal transcription factors & 3 & 0.042039395 \\
\hline
\end{tabular}

and SPRY2 (38)]. These results suggested that the present miRNA target prediction is reliable.

GO annotation analysis of miRNA targets. These 320 miRNA target genes were subjected to GO enrichment analysis. All these genes were categorized based on BP, MF and CC (Table II). In the CC category, the nucleus term was the most significant term (with the lowest P-value) and contained the largest number of genes. In the MF category, the term with the lowest P-value was transcription regulatory activity. In the BP category, the most significantly enriched genes belonged to the cell cycle and proliferation process, and a total of 42 genes were categorized to this process. These 42 genes belonged to the targets of 16 miRNAs (Table I). KEGG pathway analysis also showed similar results, with the number of genes involved in the cell cycle being the largest (Table III). This suggested that the targets of p53-related miRNAs mainly play roles in the cell cycle and proliferation process.

Network analysis of miRNA targets. To understand the association between these miRNA targets, the KEGG dataset, PPI and Pubmed datasets were integrated to construct a network 
A

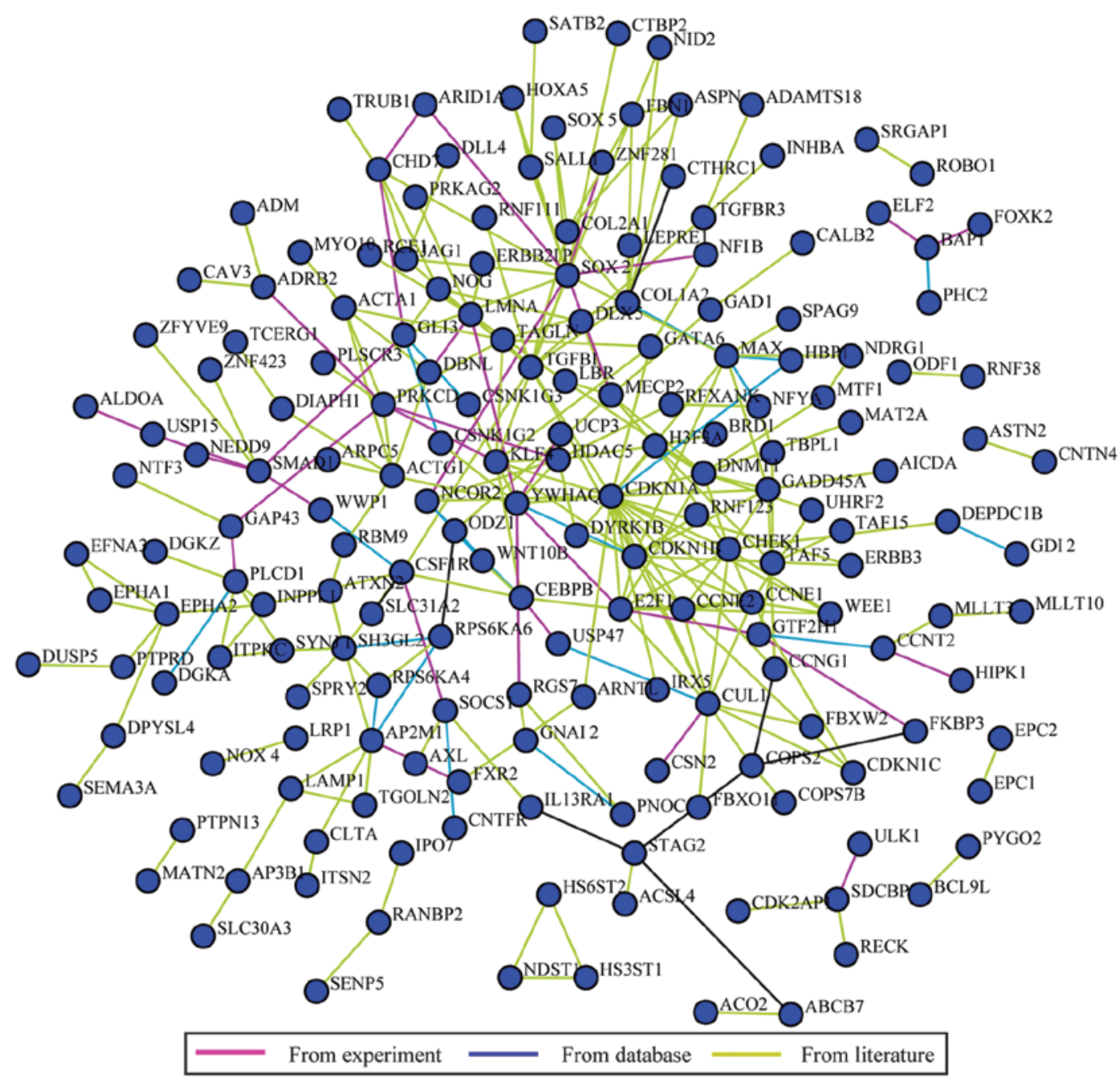

B

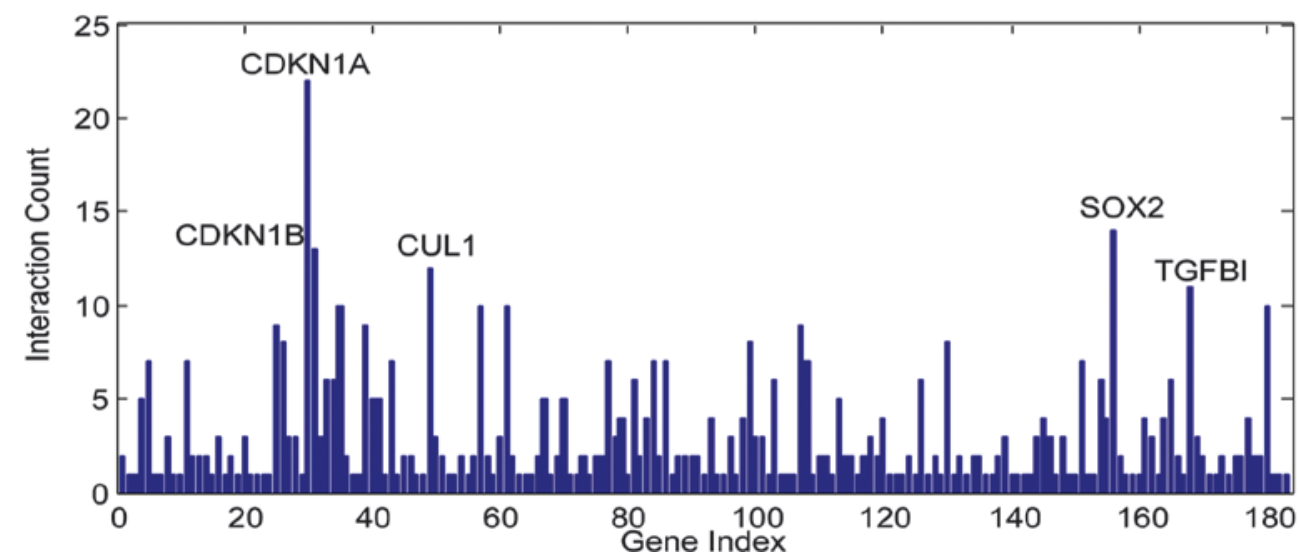

Figure 2. Network analysis of miRNA targets. (A) A network was constructed by integrating three types of data (Kyoto Encyclopedia of Genes and Genomes database, high-throughout protein interaction experiments and the literature data), and mapped in a network structure using Medusa. Each node corresponds to a gene and an edge indicates a direct interaction. (B) Interaction gene counts for microRNA target genes.

of miRNA targets (Fig. 2A). The resulting network was composed of nodes (genes) and edges (interactions). Fig. 2B shows the degree (i.e., the number of edges emanating from a node) of each node. In the present network, the nodes with degree $>10$ were defined as hubs, including CDKN1A, SOX2, CDKN1B, CUL1 and TGFBI. According to the aforementioned GO annotation, these hub genes, with the exception of TGFBI, were annotated to the term 'cell cycle and proliferation' (Table I). In a molecular interaction network, hubs are more essential for the global network structure than non-hubs (39). Therefore, it indicates the roles of targets of p53-related miRNAs in the cell cycle and proliferation, in accordance with the aforementioned pathway analysis.

\section{Discussion}

In the present study, for the first time, the interactions of miRNAs and p53 were systematically analyzed in breast 
cancer using NLP analysis, and 22 miRNAs associated with p53 in breast cancer were identified. Among these miRNAs, 11 are transcriptionally or post-transcriptionally upregulated by p53 [miR-10b $(11)$, miR-16 $(13,18)$, miR-22 (12), miR-26a (13), miR-34a (14), miR-145(18,19), miR-148a (15), miR-200b (16), miR-200c (16), miR-203 (20) and miR-205 (17)], one directly targets p53 [miR-504 (9)], and others do not directly interact with p53, but indirectly play roles in the p53 signaling pathway [e.g., miR-9 (40), miR-21 (41) and miR-222 (10)]. Bioinformatics analysis identified 320 targets of p53-related miRNAs.

Although these 22 p53-related miRNAs have different sets of targets, GO annotation revealed that the majority of the miRNA targets were significantly enriched in the cell cycle and proliferation process. In the network of miRNA targets, the five hub genes, with the exception of TGFBI, were annotated to cell cycle processes. TGFBI has been recently reported to affect the cell cycle via the regulation of $\mathrm{p} 21$ and p53 expression (42). Cyclin-dependent kinase inhibitor 1A (CDKN1A; also known as p21, Cip1 or WAF1) was identified as the most highly connected hub gene. CDKN1A has been proven to be a direct target of the p53 tumor suppressor and to play key roles in mediating p53-dependent cell cycle arrest in response to DNA damage. In a molecular interaction network, hubs are more essential for the global network structure than non-hubs (39). The results suggest that p53-related miRNAs play roles in the cell cycle. A number of studies have demonstrated that p53 acts as a key regulator of the cell cycle, mainly by transcriptional regulation of certain key genes in the cell cycle, such as CDKN1A. The present study suggested that, in addition to transcriptionally regulating cell cycle-related genes, p53 also indirectly regulates them through miRNAs. These results also suggest a previously unknown mechanism for p53 function, and thus provide an important contribution to our knowledge of p53. Furthermore, the results of the present study were consistent with those of Otsuka et al (43) which revealed that $\mathrm{p} 53$-induced miRNAs control the cell cycle and cell survival via the repression of cell-cycle regulators and/ or antiapoptotic proteins. Additionally, Rokavec et al (44) summarized previously published data regarding the interaction between p53 and miRNAs in gastrointestinal cancer, and found that a total of 32 p53-related miRNAs exhibit differential expression between normal and tumor tissue and are associated with clinical and pathological parameters of gastrointestinal cancer. Among the 32 miRNAs, only 9 miRNAs (miR-34a, miR-200a, miR-200b, miR-200c, miR-205, miR-145, miR-16, miR-22, miR-504) are common in both gastrointestinal cancer and breast cancer (Table I). Thus, we hypothesize that $\mathrm{p} 53$ regulates different sets of miRNAs in various types of cancer.

\section{Acknowledgements}

The authors would like to thank Shanghai Sensichip Infoteck Co., Ltd., (Shanghai, China) for assistance with the bioinformatics analysis. This study was supported by the National Natural Science Foundation of China (grant nos. 81071656 and 81272318) and the Cooperative Innovation Center of Engineering and New Products for Developmental Biology of Hunan (grant no. 20134486).

\section{References}

1. Riley T, Sontag E, Chen P and Levine A: Transcriptional control of human p53-regulated genes. Nat Rev Mol Cell Biol 9: 402-412, 2008.

2. Levine AJ and Oren M: The first 30 years of p53: Growing ever more complex. Nat Rev Cancer 9: 749-758, 2009.

3. Petitjean A, Mathe E, Kato S, Ishioka C, Tavtigian SV, Hainaut P and Olivier M: Impact of mutant p53 functional properties on TP53 mutation patterns and tumor phenotype: Lessons from recent developments in the IARC TP53 database. Hum Mutat 28: 622-629, 2007.

4. Gasco M, Shami S and Crook T: The p53 pathway in breast cancer. Breast Cancer Res 4: 70-76, 2002.

5. Lacroix M, Toillon RA and Leclercq G: p53 and breast cancer, an update. Endocr Relat Cancer 13: 293-325, 2006.

6. Krell J, Frampton AE, Colombo T, Gall TM, De Giorgio A Harding V, Stebbing J and Castellano L: The p53 miRNA interactome and its potential role in the cancer clinic. Epigenomics 5: 417-428, 2013.

7. Wu N, Lin X, Zhao X, Zheng L, Xiao L, Liu J, Ge L and Cao S: MiR-125b acts as an oncogene in glioblastoma cells and inhibits cell apoptosis through p53 and p38MAPK-independent pathways. Br J Cancer 109: 2853-2863, 2013.

8. Liu Y, Xing R, Zhang X, Dong W, Zhang J, Yan Z, Li W, Cui J and $\mathrm{Lu}$ Y: miR-375 targets the p53 gene to regulate cellular response to ionizing radiation and etoposide in gastric cancer cells. DNA Repair (Amst) 12: 741-750, 2013.

9. Ford NA, Dunlap SM, Wheatley KE and Hursting SD: Obesity, independent of 553 gene dosage, promotes mammary tumor progression and upregulates the p53 regulator microRNA-504. PLoS One 8: e68089, 2013.

10. Zhang C, Zhang J, Zhang A, Wang Y, Han L, You Y, Pu P and Kang C: PUMA is a novel target of miR-221/222 in human epithelial cancers. Int J Oncol 37: 1621-1626, 2010.

11. Bisio A, De Sanctis V, Del Vescovo V, Denti MA, Jegga AG, Inga $\mathrm{A}$ and Ciribilli Y: Identification of new p53 target microRNAs by bioinformatics and functional analysis. BMC Cancer 13: 552, 2013.

12. Lin J, Huo R, Xiao L, Zhu X, Xie J, Sun S, He Y, Zhang J, Sun Y, Zhou Z, et al: A novel p53/microRNA-22/Cyr61 axis in synovial cells regulates inflammation in rheumatoid arthritis. Arthritis Rheumatol 66: 49-59, 2013

13. Lezina L, Purmessur N, Antonov AV, Ivanova T, Karpova E, Krishan K, Ivan M, Aksenova V, Tentler D, Garabadgiu AV, et al: miR-16 and miR-26a target checkpoint kinases Weel and Chk1 in response to 533 activation by genotoxic stress. Cell Death Dis 4: e953, 2013.

14. Léveillé N, Elkon R, Davalos V, Manoharan V, Hollingworth D, Oude Vrielink J, le Sage C, Melo CA, Horlings HM, Wesseling J, et al: Selective inhibition of microRNA accessibility by RBM38 is required for p53 activity. Nat Commun 2: $513,2011$.

15. Xu X, Fan Z, Kang L, Han J, Jiang C, Zheng X, Zhu Z, Jiao H, Lin J, Jiang K, et al: Hepatitis B virus X protein represses miRNA-148a to enhance tumorigenesis. J Clin Invest 123: 630-645, 2013

16. Kim T, Veronese A, Pichiorri F, Lee TJ, Jeon YJ, Volinia S, Pineau P, Marchio A, Palatini J, Suh SS, et al: p53 regulates epithelial-mesenchymal transition through microRNAs targeting ZEB1 and ZEB2. J Exp Med 208: 875-883, 2011.

17. Piovan C, Palmieri D, Di Leva G, et al: Oncosuppressive role of p53-induced miR-205 in triple negative breast cancer. Mol Oncol 6: 458-472, 2012.

18. Suzuki HI, Yamagata K, Sugimoto K, Iwamoto T, Kato S and Miyazono K: Modulation of microRNA processing by $\mathrm{p} 53$. Nature 460: 529-533, 2009.

19. Spizzo R, Nicoloso MS, Lupini L, Lu Y, Fogarty J, Rossi S, Zagatti B, Fabbri M, Veronese A, Liu X, et al: miR-145 participates with TP53 in a death-promoting regulatory loop and targets estrogen receptor-alpha in human breast cancer cells. Cell Death Differ 17: 246-254, 2010.

20. Chang J, Davis-Dusenbery BN, Kashima R, et al: Acetylation of p53 stimulates miRNA processing and determines cell survival following genotoxic stress. EMBO J 32: 3192-3205, 2013.

21. Gao W, Xu J, Liu L, Shen H, Zeng H and Shu Y: A systematic-analysis of predicted miR-21 targets identifies a signature for lung cancer. Biomed Pharmacother 66: 21-28, 2012. 
22. Tang J, Zhang ZH and Liu GL: A systematic analysis of the predicted human La protein targets identified a hepatitis B virus infection signature. J Viral Hepat 20: 12-23, 2013.

23. Settles B: ABNER: An open source tool for automatically tagging genes, proteins and other entity names in text. Bioinformatics 21: 3191-3192, 2005.

24. Krek A, Grün D, Poy MN, Wolf R, Rosenberg L, Epstein EJ, MacMenamin P, da Piedade I, Gunsalus KC, et al: Combinatorial microRNA target predictions. Nat Genet 37: 495-500, 2005

25. John B, Enright AJ, Aravin A, Tuschl T, Sander C and Marks DS: Human MicroRNA targets. PLoS Biol 2: e363, 2004.

26. Lewis BP, Burge CB and Bartel DP: Conserved seed pairing, often flanked by adenosines, indicates that thousands of human genes are microRNA targets. Cell 120: 15-20, 2005.

27. Salomonis N, Hanspers K, Zambon AC, Vranizan K, Lawlor SC, Dahlquist KD, Doniger SW, Stuart J, Conklin BR and Pico AR: GenMAPP 2: New features and resources for pathway analysis. BMC Bioinformatics 8: 217, 2007.

28. Pagel P, Kovac S, Oesterheld M, Brauner B, Dunger-Kaltenbach I, Frishman G, Montrone C, Mark P, Stümpflen V, Mewes HW, et al: The MIPS mammalian protein-protein interaction database. Bioinformatics 21: 832-834, 2005.

29. Pavlopoulos GA, Hooper SD, Sifrim A, Schneider R and Aerts J: Medusa: A tool for exploring and clustering biological networks. BMC Res Notes 4: 384, 2011.

30. Wu Y, Xiao Y, Ding X, Zhuo Y, Ren P, Zhou C and Zhou J: A miR-200b/200c/429-binding site polymorphism in the 3 ' untranslated region of the AP- $2 \alpha$ gene is associated with cisplatin resistance. PLoS One 6: e29043, 2011.

31. Witkos TM, Koscianska E and Krzyzosiak WJ: Practical Aspects of microRNA Target Prediction. Curr Mol Med 11: 93-109, 2011.

32. Yang S, Banerjee S, Freitas A, Cui H, Xie N, Abraham E and Liu G: miR-21 regulates chronic hypoxia-induced pulmonary vascular remodeling. Am J Physiol Lung Cell Mol Physiol 302: L521-L529, 2012.

33. Dellago H, Preschitz-Kammerhofer B, Terlecki-Zaniewicz L, Schreiner C, Fortschegger K, Chang MW, Hack1 M, Monteforte R, Kühnel H, Schosserer M, et al: High levels of oncomiR-21 contribute to the senescence-induced growth arrest in normal human cells and its knock-down increases the replicative lifespan. Aging Cell 12: 446-458, 2013.
34. Marquez RT, Wendlandt E, Galle CS, Keck $\mathrm{K}$ and McCaffrey AP: MicroRNA-21 is upregulated during the proliferative phase of liver regeneration, targets Pellino-1, and inhibits NF-kappaB signaling. Am J Physiol Gastrointest Liver Physiol 298: G535-G541, 2010.

35. Trohatou O, Zagoura D, Bitsika V, Pappa KI, Antsaklis A, Anagnou NP and Roubelakis MG: Sox2 suppression by miR-21 governs human mesenchymal stem cell properties. Stem Cells Transl Med 3: 54-68, 2014.

36. Chen B, Chen X, Wu X, Wang X, Wang Y, Lin TY, Kurata J, Wu J, Vonderfecht S, Sun G, et al: Disruption of microRNA-21 by TALEN leads to diminished cell transformation and increased expression of cell-environment interaction genes. Cancer Lett 356: 506-516, 2014.

37. Sandhir R, Gregory E and Berman NE: Differential response of miRNA-21 and its targets after traumatic brain injury in aging mice. Neurochem Int 78: 117-121, 2014.

38. Zhao J, Tang N, Wu K, Dai W, Ye C, Shi J, Zhang J, Ning B, Zeng X and Lin Y: MiR-21 simultaneously regulates ERK1 signaling in HSC activation and hepatocyte EMT in hepatic fibrosis. PLoS One 9: e108005, 2014.

39. He X and Zhang J: Why do hubs tend to be essential in protein networks? PLoS Genet 2: e88, 2006.

40. Hsu PY, Deatherage DE, Rodriguez BA, Liyanarachchi S, Weng YI, Zuo T, Liu J, Cheng AS and Huang TH: Xenoestrogen-induced epigenetic repression of microRNA-9-3 in breast epithelial cells. Cancer Res 69: 5936-5945, 2009.

41. Frankel LB, Christoffersen NR, Jacobsen A, Lindow M, Krogh A and Lund AH: Programmed cell death 4 (PDCD4) is an important functional target of the microRNA miR-21 in breast cancer cells. J Biol Chem 283: 1026-1033, 2008.

42. Li B, Wen G, Zhao Y, Tong J and Hei TK: The role of TGFBI in mesothelioma and breast cancer: Association with tumor suppression. BMC Cancer 12: 239, 2012.

43. Otsuka K and Ochiya T: Genetic networks lead and follow tumor development: microRNA regulation of cell cycle and apoptosis in the p53 pathways. Biomed Res Int 2014: 749724, 2014.

44. Rokavec M, Li H, Jiang L and Hermeking H: The p53/ microRNA connection in gastrointestinal cancer. Clin Exp Gastroenterol 7: 395-413, 2014. 